Trinity University

Digital Commons @ Trinity

Psychology Faculty Research

Psychology Department

$12-2007$

\title{
Impairments in Inhibition or Cognitive Control in Psychological Disorders
}

Paula T. Hertel

Trinity University, phertel@trinity.edu

Follow this and additional works at: https://digitalcommons.trinity.edu/psych_faculty

Part of the Psychology Commons

Publication Details

Applied and Preventive Psychology

\section{Repository Citation}

Hertel, P.T. (2007). Impairments in inhibition or cognitive control in psychological disorders. Applied and Preventive Psychology, 12(3), 149-153. doi: 10.1016/j.appsy.2007.09.006

This Article is brought to you for free and open access by the Psychology Department at Digital Commons @ Trinity. It has been accepted for inclusion in Psychology Faculty Research by an authorized administrator of Digital Commons@ Trinity. For more information, please contact jcostanz@trinity.edu. 


\title{
Impairments in inhibition or cognitive control in psychological disorders
}

\author{
Paula T. Hertel* \\ Department of Psychology, Trinity University, 1 Trinity Place, San Antonio, TX 78212, USA
}

\begin{abstract}
Contributions to this special issue of Applied \& Preventive Psychology richly elucidate connections between a variety of psychological disorders and performance in a number of tasks that are used to reason about inhibitory deficits. This commentary calls attention to the different uses of the concept of inhibition - vernacular, neural, operational, and theoretical - and suggests that the term cognitive control avoids claims about dampened memory representations that are difficult to support. Central findings from the reviews are summarized; evidence concerning suppression-induced forgetting is featured and directions to foster application are discussed.
\end{abstract}

(C) 2007 Elsevier Ltd. All rights reserved.

Keywords: Cognitive control; Inhibition; Psychological disorders

Inhibition is a seductive construct in psychological research, particularly in accounting for phenomena associated with emotionally disordered and thought-disordered states. Cycles of rumination, implicated in problems with mood regulation, could be banished with a healthy inhibition construct. Intrusive thoughts and flashbacks, characteristic of dissociative states, could be reigned in. Clang associations and frenetic behavior might be organized anew. As scientists interested in psychological disorders, we must pay careful attention to the phenomena associated with the inhibition construct because they constitute a salient set of cognitive difficulties experienced in disordered states. As scientists interested in building parsimonious theoretical frameworks, however, we should ask whether the construct of inhibition is warranted, or whether instead we can make due with more general accounts of the same cognitive difficulties. The authors of the contributions to this special issue have summarized empirical research, the importance of which does not depend on our choice of theoretical constructs. Before offering brief comments on these important findings, I call attention to the diversity of ways in which the term inhibition has been used; inhibition is not just a theoretical construct anymore. Next, I summarize non-inhibitory approaches to explaining the phenomena.

\footnotetext{
* Tel.: +1 210999 8380; fax: +1 2109998386.

E-mail address: phertel@trinity.edu.
}

\section{Introductory notes on terminology}

\subsection{Inhibition: A term with many uses}

In the first article of this issue, Dillon and Pizzagalli begin by reminding us that the "inhibition of irrelevant or inappropriate actions, thought, and emotions is critical to adaptive functioning." By using the vernacular meaning of inhibition, indeed the importance of stopping cannot be overemphasized. And as all the contributions have illustrated, psychologically disordered people have various difficulties in bringing actions, thoughts and emotions to a halt. On the level of brain mechanisms, the concept of inhibition refers to neurochemical events, critical to perception and action; however, although chemical disturbances sometimes characterize psychological disorders, inhibition at the cellular level is typically viewed as irrelevant to cognitive psychological accounts that stress inhibition (see MacLeod, Dodd, Sheard, Wilson, \& Bibi, 2003).

An operational definition of the concept inhibition as it applies to performance in cognitive tasks refers to belowbaseline performance. That is, if some action, thought or emotion is inhibited it is less likely (or takes longer) to occur relative to a control condition. Paradigms that operationalize inhibition in this way include negative priming and suppression-induced forgetting. The distinctions between these operational uses of the term and the theoretical uses that sometimes accompany them are often blurred. Theoretically, the term inhibition is situated within the larger context of a representational model of mind. As a consequence of cognitive processing, this framework assumes 
that mental representations of percepts or concepts are activated or inhibited, for a period ranging from milliseconds to years (see Kolers \& Roediger, 1984) and with consequences for subsequent processing.

All four ways in which the term inhibition can be used vernacular, neural, operational and theoretical - can be found in the Dillon and Pizzagalli's introduction to this issue. The three reviews of disorder-related findings are also sensitive to these definitional issues. Yet it is sometimes difficult for the less sophisticated reader to realize that the use of the term in one sense is separable from the other meanings of the term. For example, all three reviews discuss evidence gathered from negative-priming paradigms. The name of the paradigm itself tends to blur the lines between operational and theoretical definitions. Negative priming can be thought about in operational terms as the increase in the time to respond on trial $n$ if the stimulus was ignored on trial $n-1$. But of course priming primarily refers to a change in the level of activation associated with a memory representation; thus, the label biases the type of explanation. In short, the multiple uses of the term inhibition sometimes make it difficult to separate the observed phenomena from the neuroscientific and theoretical accounts that are proposed to explain them.

\subsection{Alternative accounts of the phenomena}

The reviews in this issue describe non-inhibitory accounts of the observed phenomena-accounts that do not refer to the theoretical dampening of memory representations (e.g., episodic retrieval and feature-mismatch accounts of negative priming). To briefly illustrate these accounts we might say the following: a current display recruits a prior-processing episode along one or more dimensions of similarity, including time. Details of the prior-processing episode - such as attentional disengagement from the irrelevant stimulus - tend to be replicated in the current trial and thereby introduce conflict and associated cost when the task demands competing thoughts or actions. Neill and Mathis (1998) coined the term transfer-inappropriate processing to represent this mismatch of the required processes across episodes. (It is important to note that the term episodic retrieval does not imply uses of memory as a separate "entity" from other processes; instead the term is intended to stress the dependence of performance on the prior-processing episode.) Similar accounts, many of which are summarized by MacLeod et al. (2003), have been offered for performance costs in a variety of paradigms, without invoking the notion that a memory representation has been inhibited.

Is inhibition a theoretical construct that carries its own weight? The way to decide, of course, is to test hypotheses generated specifically for that purpose (see Levy \& Anderson, 2002). The way not to decide is to use the various meanings of inhibition interchangeably. As alternatives to the theoretical construct of inhibition, terms associated with cognitive control are more theoretically neutral and apply to actions as well as to thoughts. A recent essay on automaticity by Moors and De Houwer (2006) argued that the stopping of action or thought is a marker of cog- nitive control; in this broad sense, there is often no need to posit the inhibition of a particular representation (also see Moors and De Houwer for a discussion of distinctions among the concepts controlled, intentional and conscious; for example, a controlled procedure can be invoked automatically). An emphasis on cognitive control adequately addresses performance on interference tasks that are sensitive to individual differences (e.g., Kane \& Engle, 2003). And deficits in cognitive control clearly characterize performance by psychologically disordered individuals, not only in stopping thoughts and actions, but also in their initiation (see Hertel, 2000). Moreover, neuroscientific investigations identify frontal structures and functions that correspond to cognitive control. According to Dillon and Pizzagalli's introduction, measures taken during antisaccade, go/no-go and stop-signal tasks, all paradigms of response stopping that are sensitive to neural deficits, reveal likely pathways from control centers in the prefrontal cortex to more stimulus- or response-specific areas of the brain. Joormann, Yoon, and Zetsche discuss these pathways with reference to depression and note studies in which the maturation of frontal structures is correlated with improved cognitive control. Thus, the language of control provides the more general approach and one that subsumes processes involved in a variety of procedures (e.g., search, disengagement, switching, suppression, strategy use).

\section{Deficient control in psychopathological states}

The articles in the special issue share a first-order emphasis on experimental approaches to understanding deficient cognitive control in disordered states. This emphasis contrasts with the tendency for some clinical investigators to use self-report inventories to describe cognitive problems. Self-reports might be informative to the extent that individuals notice features of their own mental experience, even though they are influenced by demand, response bias and context of administration. But even when we are able to notice broad-brush tendencies to think in particular ways, the inventories themselves can offer no clues about fundamental mechanisms, because we cannot observe our own basic processes. On the other hand, while expressing similar concerns about self-reports, Joormann et al. illustrate how they can be used effectively. For example, the finding that self-reports of rumination are correlated with experimental evidence of deficient control provides converging evidence concerning the possible role of rumination in depression (also see Whitmer \& Banich, 2007). Slightly different difficulties exist when trying to reason from deficits on neuropsychological tests, such as the Wisconsin Card Sorting Task because they were not designed to reveal the operation of specific procedures, as Dillon and Pizzagalli note. Yet evidence of impairments on these tests sometimes provides direction for experimental investigations. Of course, all contributions to the special issue refer to performance on self-reporting inventories as a method for representing individual differences in psychopathological tendencies; diagnostic criteria rely on self-reports, as well. However, hypotheses about the corresponding deficits in inhibition and control are tested mainly with experimental methodologies, not merely by asking participants to report their difficulties 
in controlling or inhibiting their actions, thoughts, or emotions.

\subsection{Dillon and Pizzagalli: Evidence from neuroscience}

The introduction by Dillon and Pizzagalli is focused on differentiating neural pathways corresponding to the control of action, thought, or emotion in general terms, irrespective of psychopathology. Much of this and similar research does not demand the use of inhibition as an explanatory construct. For example, we have known for some time that depression is associated with changes in activity in prefrontal cortical regions associated with cognitive-control functions (see the review by Levine, Heller, Mohanty, Herrington, \& Miller, 2007). Another example concerns evidence for reduced hippocampal volume as a risk factor for Post Traumatic Stress Disorder. According to Dillon and Pizzagalli, this evidence suggests an impaired ability to control the expression of fear. They remind us that spontaneous recovery of previously extinguished fear responses in non-human species reveals that fear is inhibited (at least in the operational sense) instead of being "unlearned" during extinction trials. Classic theories of conditioning referred to inhibition in the theoretical sense to account for spontaneous recovery. In contrast, Dillon and Pizzagalli report evidence that fear is regulated through control mechanisms that differentially involve the amygdala and hippocampus. Moreover, some of this research on fear extinction targets inhibition at the neural level. Finding possible connections between neural inhibition and cognitive control is an important step in theory development, with implications for pharmacological approaches to psychopathology.

\subsection{Joormann, Yoon and Zetsche: Depression}

In the review of research on deficient cognitive control in depression, Joormann et al. emphasize the link between ruminative tendencies and performance in experimental tasks. They summarize ways in which the inhibition construct has been integrated into accounts of a wide variety of cognitive procedures during the past 20 years or so. Notably, the review spans evidence of deficits and biases, both typical of depression-related findings. In line with their emphasis on the role of rumination in depression, Joormann et al. use the inhibition construct to represent difficulty in disengaging from prior mental events; control functions associated with disengagement are situated at the center of depression-related differences. Clearly, the constructs disengagement and inhibition play similar roles, regardless of whether one thinks about turning away from an irrelevant stimulus on a preceding trial in a negative-priming task or from a subset of items from the previous display in the modified Sternberg task. The difference is that disengagement refers to an attentional process whereas inhibition refers to the state of a memory representation.

Perhaps the most important feature of Joormann et al.'s review is the consistent emphasis on personally relevant emotional events as central features of problems with cognitive inhibition or control. Emotion motivates ruminative thoughts at the outset, and emotion is exacerbated by the difficulty in ignoring those thoughts as a result. Also important is the corresponding brief review of neuroscientific research concerning the connections among emotional meaning, attentional processes, and emotion regulation in depressed states.

\subsection{Dorahy: Dissociative identity disorder (DID)}

Emotion is also featured in Dorahy's contribution to the special issue. It begins by describing experiments conducted within the framework of the flanker paradigm for investigating negative priming and reviews potentially related research from other paradigms. The focal result is the failure of DID participants to show the typical negative-priming effect (i.e., longer times to classify the target if it had served as distractor in the previous display) on trials preceded by a brief display of a negative word (e.g., hell), even though they produced intact negative priming on trials preceded by a neutral word (e.g., book). Unique to DID, this pattern was not found in samples of individuals with Generalized Anxiety Disorder or depression. Dorahy argues that weakened inhibition is adaptive in threat-related situations for people who have experienced repeated traumatic events. There are two alternative explanations from the point of view of transfer-inappropriate processing (Neill \& Mathis, 1998).

First, as Dorahy clearly explains, anxiety associated with the context word might actually prevent attention to distractors on the prime trial, such that attention need not be disengaged in order to respond to the prime target; effectively the probe target on a "negative priming" trial is processed much like the probe on control trials, resulting in no negative priming. Second, anxiety might distribute attention across distractors during prime trials. If distractors are attended on trial $n$, their presentation as targets on trial $n+1$ should not produce evidence of slowing. This second alternative-seen by Dorahy and others as failed inhibition-is consistent with much of what Dorahy tells us about the phenomenon of DID (e.g., focus on irrelevant features of the environment during abuse), as well as with the literature on divided attention and working memory. The flexibility of this mechanism across contexts is, furthermore, consistent with an emphasis on disturbances in cognitive control.

\subsection{Minas and Park: Schizophrenia}

Like Dorahy, Minas and Park review findings from negativepriming paradigms. Because it is difficult to measure negative priming with verbal materials in thought-disordered individuals, spatial tasks have been used. Performance in these tasks reveals clear inverse relationships between negative priming and the degree of acute disturbance or the degree of susceptibility to thought disorder (although less clear relation to the presence of medication). Minas and Park use the spotlight metaphor of attention (see Fernandez-Duque \& Johnson, 1999) to frame their review. The diameter of the spotlight (and also the opening of the window) is conceived to be wider in individuals diagnosed with schizophrenia than in other individuals. These metaphors suggest issues for future research regarding the distribution of 
attention across the larger "space." For example, should we think in terms of less controlled focus of attention in prime displays and subsequently reduced conflict on the probe trial?

Minas and Park believe that future research should identify which aspects of psychotic syndromes are more closely aligned with inhibitory deficits. The clinical sense of the need for control or inhibition points to positive symptoms. Research that permits the examination of correlation with symptoms might indeed suggest future directions. However, evidence regarding deficient cognitive control and prefrontal dysfunction in schizophrenia is already available in a variety of tasks (e.g., Braver, Barch, \& Cohen, 1999).

\subsection{Evidence for inhibition in remembering}

Joormann et al. and Dorahy describe findings relevant to disorder-related impairments in memory tasks. One line of this research-the think/no-think (TNT) paradigm-deserves extended comment because this line is aimed directly at the problem of distinguishing between theoretical inhibition and the more general concept of cognitive control. The research concerns the effects of thought-suppression on later attempts to remember. As Joormann et al. point out, the reasoning is similar to the negative-priming paradigm; turning attention away from a concept that might come to mind is like turning attention away from a percept in a prime display; both acts should make it more difficult to comply with the subsequent instruction to attend.

Briefly, the TNT paradigm begins with a phase of learning cue-target pairs, typically words. Next, individuals practice recalling the targets when given some cues and suppressing all thoughts of the targets when given other cues, although still other cues are reserved for baseline comparison on the final test, in which all cues are presented for target recall. Suppressioninduced forgetting is found when, compared to baseline, targets are less well recalled on the final test as a function of amount of previous suppression-practice.

Some results from TNT experiments suggest that individuals who are depressed or dysphoric forget less well than nondepressed controls (see Joormann et al.). Impaired forgetting in this paradigm suggests impaired control of attention-away from targets-during the suppression phase of the experiment. However, other TNT experiments (although not with depressed samples) argue more directly for the usefulness of an inhibition construct. The critical test, invented by Anderson and Green (2001), follows the usual final test of cued recall with a second test involving study-independent cues, yet still requiring recall of studied targets. For example, if ordeal-roach is studied and ordeal is used as the cue for suppression-practice and on the first final test, insect- $r_{--}$serves as the independent cue on the second final test. Anderson and Green found evidence for suppression-induced forgetting even when targets were cued in this study-independent way. Importantly, inhibition seems to be the only way to explain why the suppressed thought is poorly recruited by episodically irrelevant information. Therefore, tests with independent cues should be used by researchers interested in building theories of psychological dis- orders around the construct of inhibition (see Wessel, Wetzels, Jelicic, \& Merckelbach, 2005, for TNT research on dissociation).

Very recently, a different type of support for the theoretical construct of inhibition was found in a neuroimaging study with faces as cues and emotional pictures as targets in the TNT paradigm. Depue, Curran, and Banich (2007) conducted hypothesis-driven scans during recall-practice, suppressionpractice and control trials. Prefrontal regions corresponding to cognitive control showed greater activation on suppression trials, compared to baseline. Also, sensory areas of the posterior cortex (during the suppression of emotional pictures) and attentional gating areas of the thalamus normally active during memory retrieval showed decreased activity on suppression trials. The hippocampus and amygdala showed similar reductions. This pattern of activation of control structures and reduction in task specific areas goes a long way to providing support for the notion of cognitive inhibition (see Anderson et al., 2004, for related findings). Importantly, during the last block of suppression attempts, hippocampal activity was reduced to the extent that the image was later forgotten on the final test. This was a later-occurring process; early attempts to suppress implicated control of sensory activity. These changes in patterns as a function of practice suggest that mechanisms of cognitive control can result in neural versions of inhibited memory representations. The later changes also correspond to our sense of inhibition as an automatic process that no longer requires the control of attention to other matters. Evidence from a related neuroimaging study, performed during a retrieval-induced forgetting procedure, also suggests that there are neural benefits to having suppressed competing memories (Kuhl, Dudukovic, Kahn, \& Wagner, 2007). Demands on the prefrontal cortex were lessoned to the degree that unpracticed but potentially competing targets were forgotten on the final test.

The changes with practice observed in these neuroimaging studies are clinically interesting because they suggest that continued attempts to suppress are qualitatively important. (They also might address the question of why findings in TNT and related experiments on the consequences of suppression seem to differ from self-reported failures in other thought-suppression paradigms in which cues are not repeatedly presented.) Finally, Depue et al. also noted the involvement of the right-hemisphere pathway from control structures to hippocampus and amygdala during tasks that involve emotion regulation; the similarity between these patterns is consistent with behavioral evidence for connections among rumination, impaired control, and emotion regulation in depression (see Joormann et al.). A next step for researchers interested in disorder-related impairments is to determine whether these activation/reduction patterns change across samples in ways that converge with our knowledge of behavioral deficits.

\section{Conclusions}

Contributions to this special issue of Applied \& Preventive Psychology richly elucidate connections between psychological disorders and performance in tasks that are used to reason about 
inhibitory deficits. In most of the reviews, connections between cognitive deficits and emotional features of the task or context play prominent roles in differentiating performance.

I conclude first with a cautionary note: we should be conservative in claiming deficits in either cognitive control or inhibition under conditions in which inattention is suspected. Dorahy raised this issue concerning evidence of reduced negative priming in DID by asking whether avoidant processing styles extend to "failed" processing styles. Minas and Park's review suggests that a similar wariness might apply to reduced negative priming in schizophrenia. Engagement is a prerequisite for disengagement. In experiments on negative priming, evidence of positive priming would help to resolve this issue. Similar issues possibly occur in relation to performance by disordered individuals in other paradigms. For example, depression-related deficits in remembering that result from impaired control during learning can masquerade as superior suppression-induced forgetting. In short, these reviews remind us that the determination of processing deficits is often not as straightforward as it might initially seem. If this is true in experiments, how much more true might it be in applied settings?

My second set of concluding comments concerns application. Consider the finding from Dorahy that negative priming did not occur in threat-related contexts in the DID group. At first, extrapolating these results to the real-world might seem difficult until we realize that these carefully controlled tasks merely make it possible to identify effects that occur ubiquitously. The connections between rumination and emotion discussed by Joormann et al. make real-world examples more obvious. The question to ask next is: how might clinical researchers facilitate the application of our knowledge about control deficits to therapeutic uses? And the answer is likely to be found in attempts to train attentional control. Research on training the control of attention-whether through mindfulness techniques or some more specific procedure-should inform both theory and practice. Again, let us consider an example from Dorahy's essay and his suggestion that divided attention provides a model for control difficulties in DID. Training attentional control and showing subsequent negative priming is a potentially useful strategy. Similarly, considering the possibility that depressed people might have difficulty suppressing unwanted thoughts, efforts to train the use of thought substitutes during suppression in the TNT paradigm provides another direction (Joormann, Hertel, LeMoult, \& Gotlib, submitted for publication). As illustrated decades ago by classic research on the extinction of fear responses in non-human animals, a useful perspective for understanding a phenomenon is one that offers an approach to its elimination (e.g., spontaneous recovery). This perspective can guide the development of beneficial procedures for overcoming control deficits, as well. Importantly, this perspective is useful for the development of both theory and practice.

Finally, although clinical practice might not initially care whether the effects described are better conceptualized as inhibition or cognitive control, theoretical progress depends on getting our terms straight. Do we always mean inhibition in the theoretical sense when we use it operationally, in the vernacular, or to describe neural processes? The contributions to the special issue all show that difficulties in stopping actions, thoughts and emotion are exacerbated in psychologically disordered states. Whether we should explain all these difficulties in terms of poorly dampened memory representations or merely dysfunctional mechanisms of control is not yet clear.

\section{References}

Anderson, M. C., \& Green, C. (2001). Suppressing unwanted memories by executive control. Nature, 410, 366-369.

Anderson, M. C., Ochsner, K., Kuhl, B., Cooper, J., Robertson, E., Gabrieli, S. W., et al. (2004). Neural systems underlying the suppression of unwanted memories. Science, 303, 232-235.

Braver, T. S., Barch, D. M., \& Cohen, J. D. (1999). Cognition and control in schizophrenia: A computational model of dopamine and prefrontal function. Biological Psychiatry, 46, 312-328.

Depue, B. E., Curran, T., \& Banich, M. T. (2007). Prefrontal regions orchestrate suppression of emotional memories via a two-phase process. Science, 317, 215-219.

Fernandez-Duque, D., \& Johnson, M. L. (1999). Attention metaphors: How metaphors guide the cognitive psychology of attention. Cognitive Science, 23, 83-116.

Hertel, P. T. (2000). The cognitive-initiative account of depression-related impairments in memory. In D. Medin (Ed.), The psychology of learning and motivation (pp. 47-71). San Diego: Academic Press.

Joormann, J., Hertel, P. T., LeMoult, J., \& Gotlib, I. H. (submitted for publication). Training forgetting of negative material in depression.

Kane, M. J., \& Engle, R. W. (2003). Working-memory capacity and the control of attention: The contributions of goal neglect, response competition, and task set to Stroop interference. Journal of Experimental Psychology: General, 132, 47-70.

Kolers, P. A., \& Roediger, H. L. (1984). Procedures of mind. Journal of Verbal Learning \& Verbal Behavior, 23, 425-449.

Kuhl, B. A., Dudukovic, N. M., Kahn, I., \& Wagner, A. D. (2007). Decreased demands on cognitive control reveal the neural processing benefits of forgetting. Nature Neuroscience, 10, 908-914.

Levine, R. L., Heller, W., Mohanty, A., Herrington, J. D., \& Miller, G. A. (2007). Cognitive deficits in depression and functional specificity of regional brain activity. Cognitive Therapy and Research, 31, 211-233.

Levy, B. J., \& Anderson, M. C. (2002). Inhibitory processes and the control of memory retrieval. Trends in Cognitive Sciences, 6, 299-305.

MacLeod, C. M., Dodd, M. D., Sheard, E. D., Wilson, D. E., \& Bibi, U. (2003). In opposition to inhibition. In B. H. Ross (Ed.), The psychology of learning and motivation (pp. 163-214). San Diego, CA: Academic Press.

Moors, A., \& De Houwer, J. (2006). Automaticity: A theoretical and conceptual analysis. Psychological Bulletin, 132, 297-336.

Neill, W. T., \& Mathis, K. M. (1998). Transfer-inappropriate processing: Negative priming and related phenomena. In D. L. Medin (Ed.), The Psychology of learning and motivation (pp. 1-44). San Diego: Academic Press.

Wessel, I., Wetzels, S., Jelicic, M., \& Merckelbach, H. (2005). Dissociation and memory suppression: A comparison of high and low dissociative individuals' performance on the Think-No Think task. Personality and Individual Differences, 39, 1461-1470.

Whitmer, A. J., \& Banich, M. T. (2007). Inhibition versus switching deficits in different forms of rumination. Psychological Science, 18, 546-552. 